\title{
HEBRÄISCHES ÜBUNGSBUCH.
}

\author{
SEPARATAUSGABE
}

FÜR DIE

BESITZER DER ERSTEN AUFLAGE DER SCHULREGELN

DER HEBRÄISCHEN GRAMMATIK

voN

\section{KARL LUDWIG}

oberlehrer aM GYMNASIUM ERNESTINUM n GOTHA.

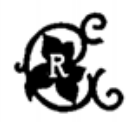

GIESSEN

J. RICKER'SCHE VERLAGSBUCHHANDLUNG 1899. 
Alle Rechte vorbehalten. 